See discussions, stats, and author profiles for this publication at: https://www.researchgate.net/publication/349501823

\title{
The Energy Spectral Density of the Mertens Function
}

Research · February 2021

CITATIONS

0

3 authors:

Darrell Cox

Grayson County College

42 PUBLICATIONS 7 CITATIONS

SEE PROFILE

Sourangshu Ghosh

Indian Institute of Technology Kharagpur

25 PUBLICATIONS 4 CITATIONS

SEE PROFILE

Some of the authors of this publication are also working on these related projects:

Reference Architecture for Pharma, Healthcare \& Life Sciences View project

New maths theorems View project
READS

46

Eldar Sultanow

Universität Potsdam

104 PUBLICATIONS 123 CITATIONS

SEE PROFILE 


\title{
The Energy Spectral Density of the Mertens Function
}

\author{
Darrell Cox, Eldar Sultanow, and Sourangshu Ghosh
}

March 21, 2021

\begin{abstract}
The Mertens function is the summatory Möbius function but the Mertens function can be generated recursively without using this definition. This recursive definition is the basis of autocorrelations that can be done on sequences of Mertens function values. Fourier transforms of the autocorrelations result in the energy spectral density. A likely upper bound of the absolute value of the Mertens function is determined.
\end{abstract}

\section{Introduction}

Lehman [1] proved that $\sum_{i=1}^{x} M(\lfloor x / i\rfloor)=1$ where $M(x)$ denotes the Mertens function. $M(x)$ is then equal to $1-\sum_{i=1}^{x-1} M(\lfloor x / i\rfloor)$. It is only necessary to compute the first third $(\lfloor x / 3\rfloor)$ of the sums - the sum of the remaining terms is $\lfloor(x+1) / 2\rfloor$.

\section{Autocorrelation}

Let $a(x)$ denote $\{M(\lfloor x / 1\rfloor), M(\lfloor x / 2\rfloor), M(\lfloor x / 3\rfloor), \ldots, M(\lfloor x / x\rfloor)\}, x=1,2,3, \ldots$ A plot of the autocorrelation of $a(x)$ for $x=50$ is 


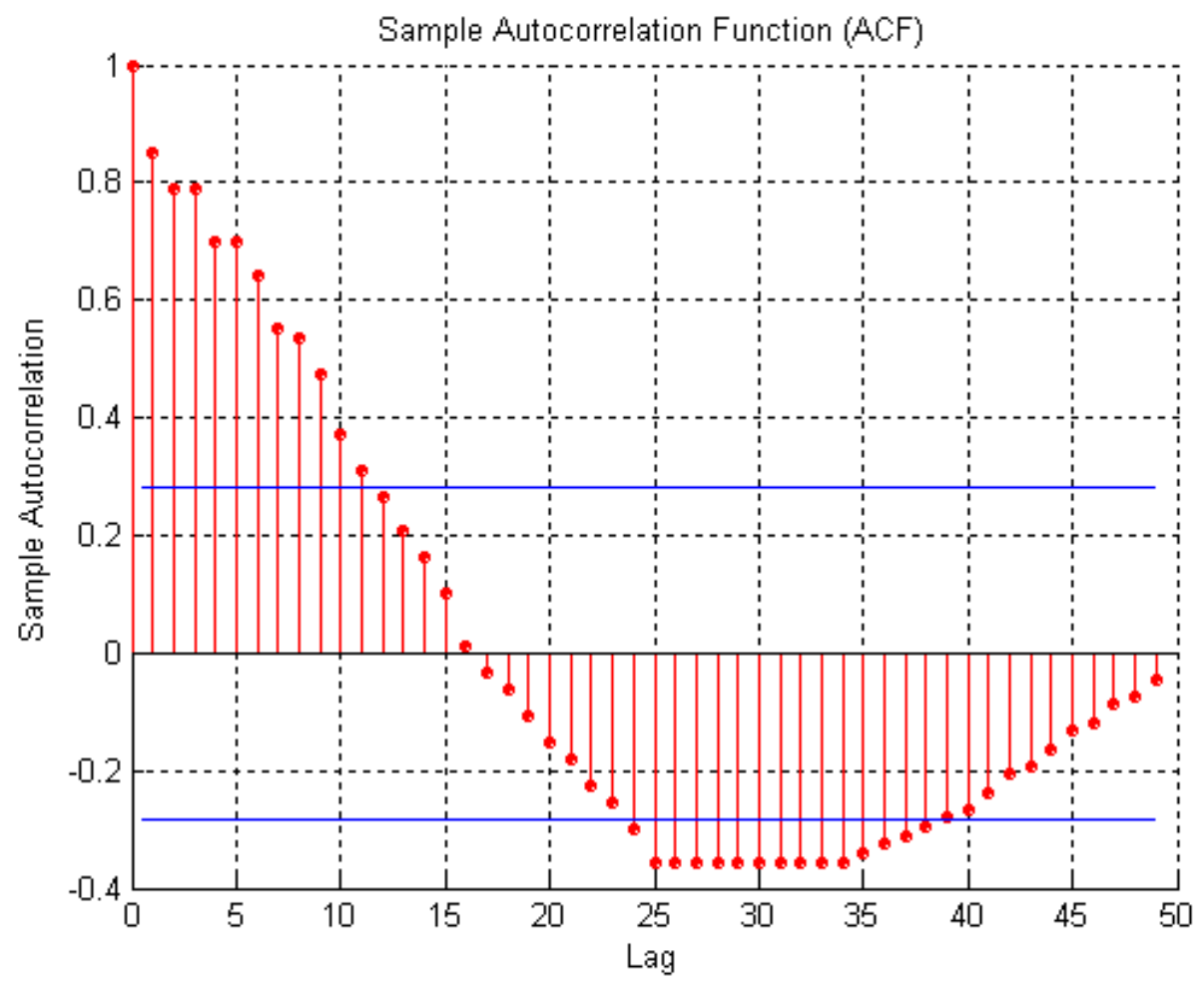

The autocorrelations for other $x$ values are similar.

\section{Fourier Transforms}

The energy spectral density is defined as $\int_{-\infty}^{\infty}|x(t)|^{2}$. The energy spectral density of $x(t)$ and the autocorrelation of $x(t)$ form a Fourier transform pair, a result known as the Wiener-Khinchin [2] [3] theorem. A plot of the moduli of the Fourier transform of the above autocorrelation is 


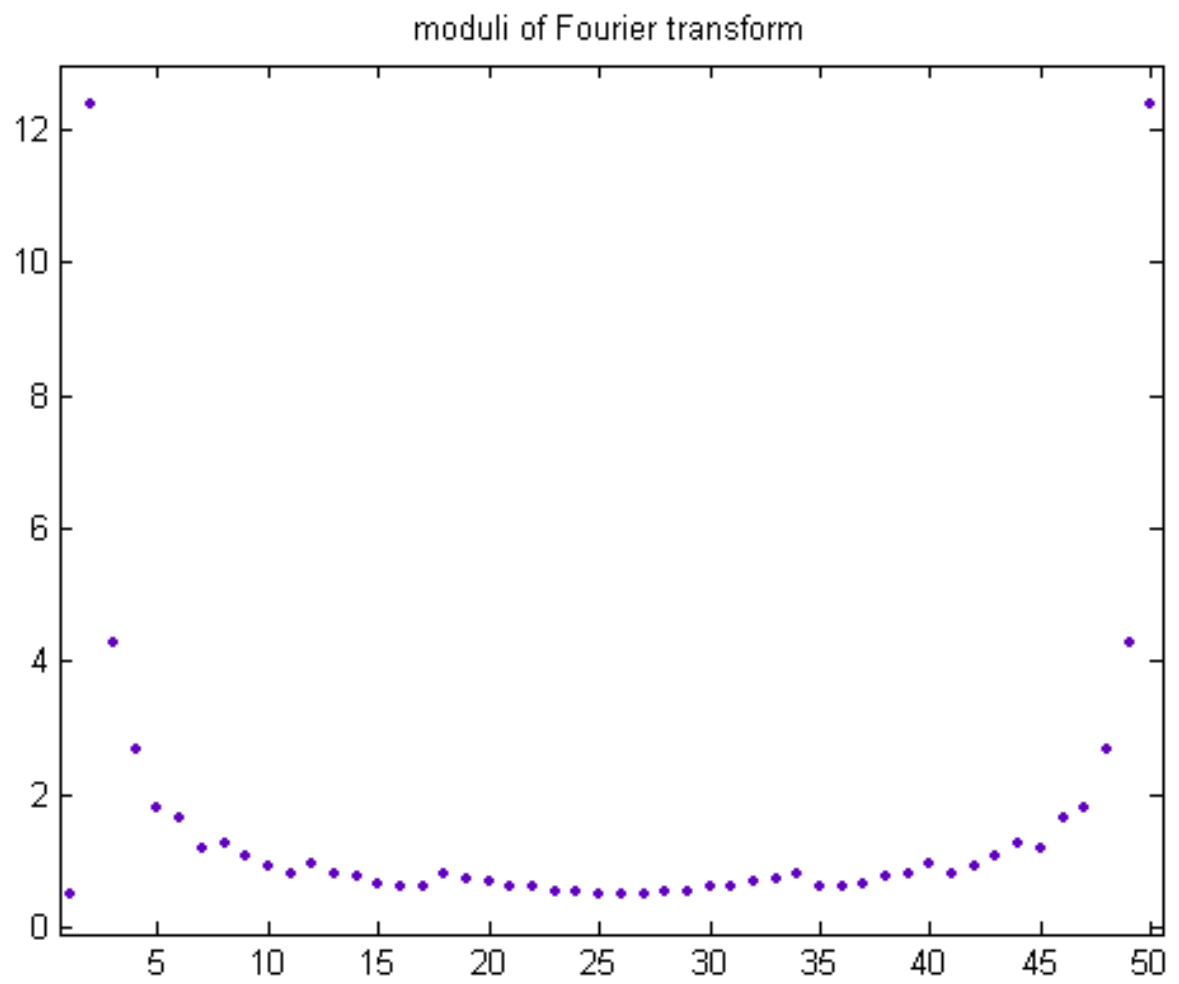

Let $s(x)$ denote the sum of the moduli (the total energy). A plot of $\sqrt{s(x)}$, $\sqrt{\log (x !) / 3.0}$, and $M(x)$ for $x=2,3,4, \ldots, 100000$ is 


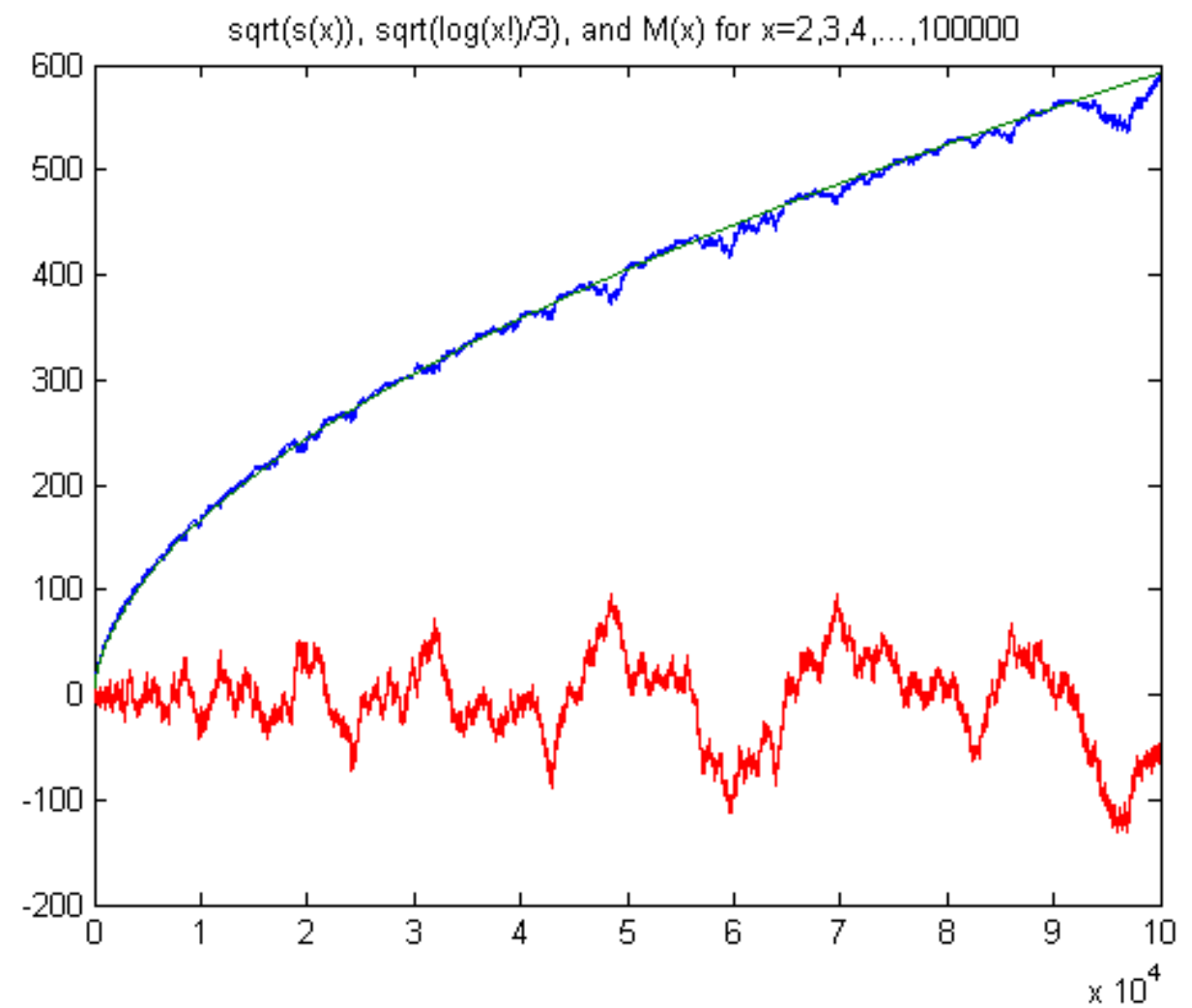

A relationship between $\sqrt{s(x)}$ and $M(x)$ is to be expected since the energy spectral density is computed using the squares of the Mertens function values. The Mertens [4] conjecture states that $\sqrt{x}$ is greater than $|M(x)|$. Odlysko and te Riele [5] proved that $\lim _{x \rightarrow \infty} \sup |M(x)| x^{-1 / 2}>1.06$ and $\lim _{x \rightarrow \infty} \inf M(x) x^{-1 / 2}<-1.009$. They say that their disproof of the Mertens conjecture can undoubtedly be used to produce larger values for $\lim _{x \rightarrow \infty} \sup |M(x)| x^{-1 / 2}$ than 1.06 with the use of more computer time and that their disproof provides some additional evidence that no such inequality of the form $|M(x)| \leq c x^{1 / 2}$ holds for any fixed $c$. They also conjecture that $\lim _{x \rightarrow \infty} \sup |M(x)| x^{-1 / 2}=\infty$. An upper bound of $\sqrt{\log (x !) / 3}$ for $|M(x)|$ is not inconsistent with this.

Cox [6] conjectured that $\log (x !)>\sum_{i=1}^{x} M(\lfloor x / i\rfloor)^{2}$ when $x>7$. By Stirling's formula, $\log (x !)=x \log (x)-x+O(\log (x))$. (Stirling's approximation of 
$\log (x !)$ is $\left(x+\frac{1}{2}\right) \log (x)-x+\frac{1}{2} \log (2 \pi)$.) Since $\log (x)$ increases more slowly than any positive power of $x$, this is a better upper bound of $\sum_{i=1}^{x} M(\lfloor x / i\rfloor)^{2}$ than $x^{1+\epsilon}$ for any $\epsilon>0$.

\section{References}

[1] Lehman, R. S., On Liouville's Function, Math. Comput. 14:311-320 (1960)

[2] Wiener, Norbert (1964), Time Series. M.I.T. Press, Cambridge, Massachusetts, p.42

[3] Khintchine, Alexander (1934), "korrelationstheorie der stationären stochastischer Prozesse", Mathematische Annalen, 109 (1): 604-615

[4] Mertens, F. (1897) Uber eine zahlentheoretische Funktion, Sitzungsberichte der Kaiserlichen Akademie der Wissenschaften, Abteilung 2a. 106: $761-830$

[5] Oklyzko, A.M. and te Riele, H.J.J. (1985), Disproof of the Mertens Conjecture, Journal für die reine und angewandte Mathematik, 357: 138-160

[6] Cox, Darrell and Ghosh, Sourangshu (2020), Bounds of the Mertens Function, 10.31219/osf.io/86whq 